\title{
Combustion Test and Numerical Analysis upon Diesel Engine
}

\author{
Yang Kun \\ Power Engineering College \\ Naval University of Engineering \\ Wuhan, China \\ e-mail: yangkundexiangzi@sina.com \\ Ouyang Guangyao \\ Power Engineering College \\ Naval University of Engineering \\ Wuhan, China \\ e-mail: 150006224@qq.com
}

\author{
Chang Yuan \\ Power Engineering College \\ Naval University of Engineering \\ Wuhan, China \\ e-mail: 504292159@qq.com

\section{Liu Qi} \\ Power Engineering College \\ Naval University of Engineering \\ Wuhan, China \\ e-mail: chyu2048@163.com
}

\begin{abstract}
It is an important way to research the IC engine combustion process by calculating the heat release rate from the cylinder pressure. Basing on the NI PXI-8196 embedded platform system, the PXI-6229 multi-function data acquisition card is put into use to realize cylinder pressure curve point sampling, and then, the combustion process heat release rate is calculated from the cylinder pressure curve data. To solve the problem of determination of the combustion starting point considering the oscillating of the heat release rate curve, a new simple and convenient method is introduced for the combustion starting determination which can make us research the combustion process more directly and exactly. Through the combustion heat release rate calculation, many more useful parameter values can be got, and the combustion starting point's determination criteria brought forward in this paper is also proofed to be very effective. The combustion heat release rate calculation method and combustion starting point criteria that is presented in this paper can be used effectively and directly, which can make a good foundation for further improvement design and research upon engine combustion system.
\end{abstract}

Key words: heat release rate; numerical analysis; point sampling; combustion; calculation;

\section{INTRODUCTION}

The cylinder pressure curve carry on lots of useful messages, which has significant meanings in thermal performance analysis v fault diagnosis and hardware-in-loop simulation ${ }^{[1]}$. But, it is difficult to diagnose the diesel engine's combustion state exhaustively only by the cylinder pressure curve. However, the calculation of the heat release rate regulation of the engine basing on the cylinder pressure curve can help us do more useful things like: analysing the influence upon the combustion process from the design parameters such as the fuel supply rate ${ }^{[2,3]}$, the spray cone angle and the shape of the combustion chamber, which can help us improve the engine's combustion process and its economic and power performance.

\section{CYLINDER PRESSURE DATA ACQUISITION}

In fact, even at a very steady working condition it will spend a very different time to rotate for the same angle because of the fluctuation of the engine crankshaft ${ }^{[4]}$. Therefore it is inaccurate to sample the pressure data by time sampling method. Nevertheless if we can realize the point sampling, then the problem will be easily resolved. Refer to the point sampling method in which the key problem is the production of the external point trigger signal. Instead of traditional low fidelity angle encoding board because of the working accuracy, the high fidelity photoelectric encoder can produce an even accurate point trigger signals to obtain a high credible cylinder pressure data.

Considering the point sampling, the external trigger and external clock source sampling should be adopted instead of the internal clock sampling. In this system the external trigger source and external clock of the hardware trigger are all provided by the photoelectric encoder. To eliminate the over frequency disturbance, a optical coupler modulation circuit is devised between the photoelectric encoder and the data acquisition card. The SY-ES40-DA720 incremental photoelectric encoder adopted in the system is difference string driving type. There are three output pulse signals, in which the A phase and the B phase differs 90 degrees and every two adjacent pulse rising edge differs 0.5 degree crankshaft. All of the two above pulse signals are produced 720 pulses per cycle, while only the third $\mathrm{Z}$ phase signal is sent out one pulse per cycle that is setted as the reference signal corresponding to some reference position. The $\mathrm{Z}$ phase signal of the photoelectric encoder is setted as the external trigger source which is connected to the PFI0 port of the 6229 data acquisition card to control the beginning of the data acquisition; the A phase signal is connected to the PFI4 port that is setted as the external clock source to control the point sampling of the cylinder pressure data. Considering the photoelectric encoder not absolute type, it 
can not indicate the position of the crankshaft angular indexing(for example: the top dead center position), which leads to the random start point making a big difficulty for the incoming cylinder combustion analysis. To resolve

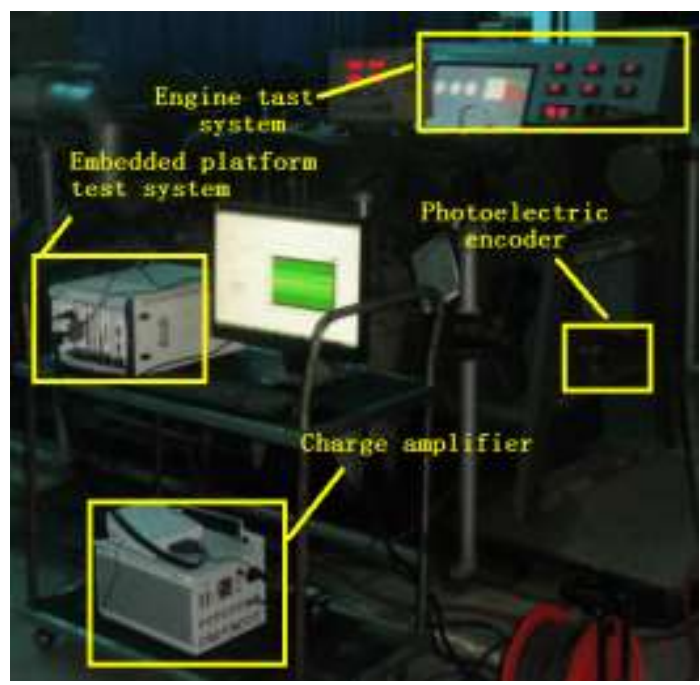

this problem, an additional magnetoelectric revolution sensor is installed at the flywheel to pick up the top dead center signal. The Fig .1 is the arrangement of the testing instruments

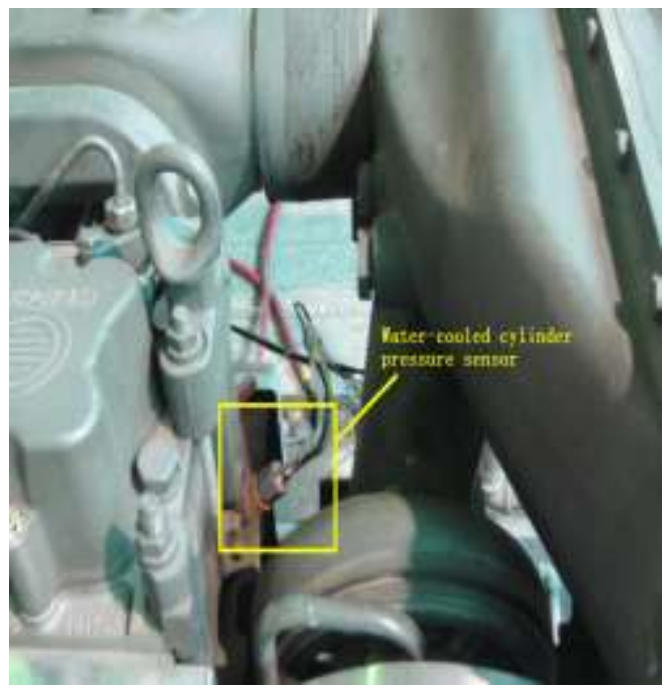

Figure 1. The arrangement of the testing instruments

It is so difficult to make arrangement for the pressure measurement passageway in the cylinder head because the WD615 diesel engine is so compact. Then after a careful wire-electrode cutting to the cylinder head, a pressure measurement passageway is successfully designed to mount the cylinder pressure sensor. The pressure sensor is a water-cooled piezo-electric sensor whose type is KISTLER 601A. The charge signal of the sensor and the top dead center signal are respectively linked to AIO and AI1 port. Finite data points sampling method is adopted, and 72,000 data points are sampled for one time. But during the data acquisition process, a serious "smear" phenomenon appears, that is the cylinder pressure signal is badly disturbed by the top dead center signal which leads to a drastic wave distortion. Finally the cause was found that the problem is owing to totally the asynchronous working mode of the data acquisition card, that is when the channel is changed the charge can not be released timely. Then to resolve this disturbance problem, a ground connection measure is taken to release the charge timely, and an unpredictable satisfactory effect was got.

\section{COMBUSTION HEAT RELEASE RATE NUMERICAL}

ANALYSIS

The working process numerical analysis of the internal combustion engine is an effective method to research the diesel's combustion process ${ }^{[5]}$. The numerical analysis of the internal cylinder working process can give comprehensive and clear assurance to the combustion performances, which makes great sense to the fault diagnosis and improvement of the combustion system.

\section{A. Multi-cycle data averaging}

The intake process injection process、 fire process and the combustion process are all very complex, which leads to a very complex variety of the in-cylinder pressure. Although the diesel engine seems to be at a steady state from the macroscopic view, the thermal state in the cylinder is not steady. So the numerical analysis based on only one cycle is obviously inaccurate. To obtain a representational pressure curve, many cycles' data are necessary. And the data averaging method is introduced in this paper to eliminate the random error that is brought about by the disproportion through different cycles. The concrete method as follows:

1) Calculation the average value of the cylinder pressure

According to the least square procedure, the arithmetic average value of the data points at any time equals to:

$$
p_{\varphi}^{m}=\sum_{j=1}^{n} p_{\varphi}^{j} / n
$$

Thereinto, $p_{\varphi}^{1}, p_{\varphi}^{2}, \Lambda p_{\varphi}^{n}-$ different cycles' cylinder pressure for the same crankshaft angle; while $n$ is the number of the cycles.

2) Calculation of the probable error $\gamma_{\varphi}$

$$
\gamma_{\varphi}=0.6745 * \sqrt{\frac{\sum_{j=1}^{n}\left(p_{\varphi}^{j}-p_{\varphi}^{m}\right)^{2}}{n-1}}
$$

3) Elimination of the doubtful pressure values When the result of the computational formula $\left|\left(p_{\varphi}^{j}-p_{\varphi}^{m}\right) / \gamma_{\varphi}\right|=\delta$ is greater than 3.8, the pressure value $p_{\varphi}^{j}$ will be eliminated.

4) Calculation the average value of the ultimate 
effective pressure points

$$
p_{\varphi}^{m}=\sum_{j=1}^{n^{\prime}} p_{j}^{\varphi} / n^{\prime}
$$

Thereinto, $n^{\prime}$ is the total number of the cycles in which the doubtful pressure value has been eliminated.

In this paper, the tested number of the cycles is 50 , and the Fig .2 is the Multi-cycle medial cylinder pressure curve

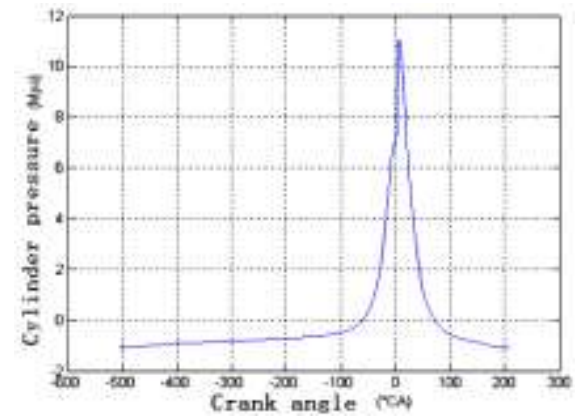

Figure 2. Multi-cycle medial cylinder pressure curve

\section{B. Cylinder pressure curve data correction}

The calculation result of the heat release rate often has a big oscillating because of the random disturbance in the cylinder pressure curve, which will cover the main property of the heat release rate curve. To diminish the disturbance, a proper correction to the pressure curve is necessary.

Suppose the cylinder's volume is $V$ when the crankshaft position is $\varphi$, then the product of the pressure and the volume correspond to the crankshaft position $\varphi$ can be easily obtained. Considering that the instantaneous temperature and mole number of the working medium is continuous, meanwhile referring to the State equation $p^{\prime} \cdot V=M R T$, it can be easily known that the $p^{\prime} \cdot V-\varphi$ data curve should be a smooth data curve. However in fact, the actual $p^{\prime} \cdot V-\varphi$ is not quite smooth because of the sampling error. In the paper, the five point three method is introduced to smooth the $p^{\prime} \cdot V-\varphi$ data curve, then the value of $p$ can be easily derived from the smooth $p^{\prime} \cdot V-\varphi$ data curve.

\section{Calibration of the cylinder pressure data}

Although the pressure sensor has been carefully calibrated before the experiment, the actual pressure appears to be negative according to the Fig .2 ahead. So the data should be calibrated to eliminate the zero point drift influence ${ }^{[6,7]}$. Moreover the top dead center position should be corrected too to remove the static error by the pure compressing line method ${ }^{[8]}$.

\section{Calculation of the heat release rate}

The working medium is supposed to be ideal gases because of its concrete working conditions ${ }^{[9,10]}$, the single area implosive combustion computational model is adopted. While the determination of the combustion starting point is so difficult that a new simple and direct criteria is put forward. The concrete steps as follows:

Firstly, a number of data points are chosen close up to the top dead center to search the combustion starting point in proper sequence, the max crankshaft angle data point that satisfy all the two requirements below is thought as the starting point:

1) all of the right-handed points' value are greater than this point's value ;

2) the maximal value of the ratio between the definite points and this point is greater than a threshold that is determined by the $80 \%$ of the max pressure rising rate numerical times.

\section{E. Heat release rate calculation result of the WD615 diesel engine}

The working condition of the diesel engine is set at the revolution of $1200 \mathrm{r} / \mathrm{min}$ and the effective power $100 \mathrm{~kW}$. The in-cylinder pressure rising rate numerical analysis result is shown at Fig .3, in which the asterisk point is the combustion starting point that is determined by the criteria above. Fig .4 and Fig .5 are heat release rate calculative result when pressure data not corrected and corrected respectively. There is a strong oscillating in the Fig .4, it is obviously that the main performance of the heat release rate curve is covered. However the Fig .5 gives us a clear trend of the heat release rate. There are many more critical parameter values can be easily obtained, such as the maximal heat release rate peak value $294.9 \mathrm{~J} /{ }^{\circ} \mathrm{CA}$ with its phase $4^{\circ} \mathrm{CA}$, the combustion end point phase is $47^{\circ} \mathrm{CA}$. Moreover it can be discovered that the whole combustion process is comparatively short, and the process ends at crank angle $50^{\circ} \mathrm{CA}$. In summary, the combustion heat release is intensive close to the top dead center, which indicates the engine has a good economical efficiency. On the other hand, the very low specific fuel consumption experimental data of $198 \mathrm{~g} / \mathrm{kWh}$ proofs that too.

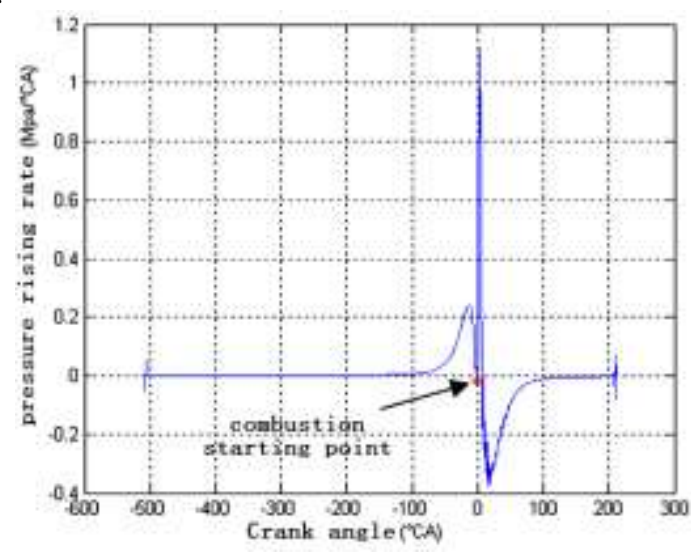

Figure 3. Engine cylinder pressure rising rate curve 


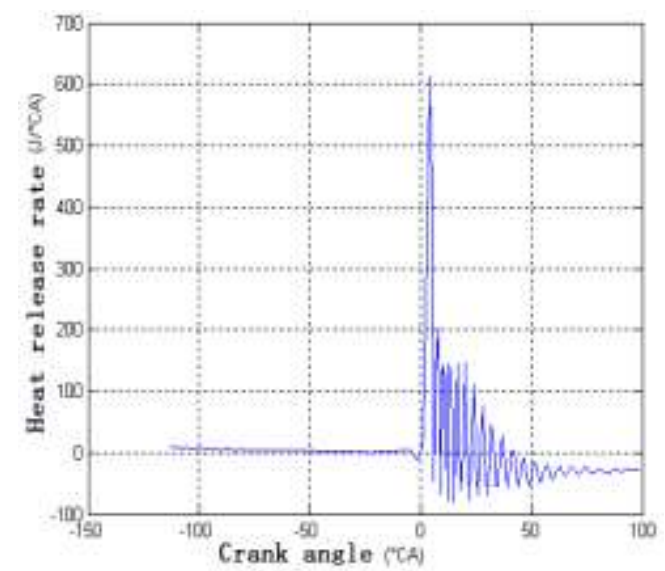

Figure 4. Heat release rate computational result(not corrected)

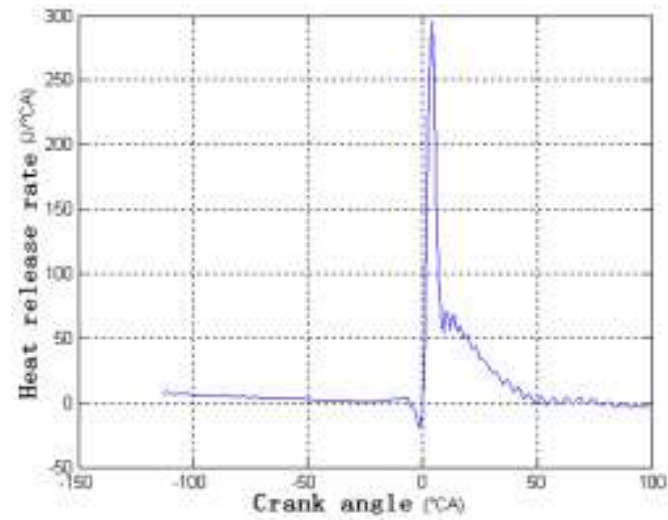

Figure 5. Heat release rate computational result(corrected)

\section{CONCLUSIONS}

An elaborative combustion analysis has been done upon the WD615 diesel engine in the paper, and then conclusions can be drawn as follows :

1) through the employment of the top dead center sensor and the optical coupler circuit, the pressure drift and the alternating current disturbance are resolved;

2 ) through the employment of multi-cycle pressure data averaging method and the pressure correction, the zero point drift and the probable error are removed;

a new simple and direct criteria to determine the combustion starting point is put forward in this paper.
Experimental data proofs that the numerical computational results of the heat release rate can reflect the actual heat release regulation objectively.

\section{ACKNOWLEDGMENT}

This work is supported by the National Natural Science Foundation of China (51409254) and the Naval University of Engineering guide Foundation (HGDYDJJ13005). We are grateful for their financial supports.

\section{REFERENCES}

[1] X. R. Li, R. Wei, B. G. Sun, W. Du, L. Zhou. Internal Combustion Engine Computing Combustion Science and Technology[M]. Beijing: Beijing University of Aeronautics and Astronautics press, 2012.

[2] M. Z. Xie. Internal Combustion Engine Computing Combustion Science.[M]. Dalian: Dalian University of Technology Press, 2005

[3] J. Zhang, G. Y. Li, Y. Yuan. Numerical Simulation and Analysis of the Influence of Fuel Injection Rate-shape on Diesel Engine Combustion Process[J]. Acta Armamentarii, Vol. 33, Mar. 2012, pp. 347-353.

[4] X. C. Wang, J. Li, M, He. Real Vehicle Test and Analysis of Combustion Process for High Power Diesel Engine in Plateaus[J].Vehicle Engine, vol. 3, Jun. 2012, pp. 32-36.

[5] S. Zhou, Y. Y. Wang, P. J. Ming, and Y. Q. Qing. Internal Combustion Engine Working Process Simulation Technology.[M] Beijing: Beijing University of Aeronautics and Astronautics press, 2012.

[6] W. Du, F. S. Liu, Z. J. Li. Experimental Study on Fluctuation of Intake Pressure in Turbocharged Diesel Engine[J]. Chinese Internal Combustion Engine Engineering, 2008, 29(3): 37-40.

[7] MICHAEL F. J, CHRISTOPHER R. Pond Evaluation of Techniques for Absolute Cylinder Pressure Correction. SAE TECHNICAL PAPER SERIES 970036.

[8] X. H. Zou, C. Chen, Y. Yang. Algorithm Design on Computing Dynamic Top Dead Center with Compressing Line Method[J]. Small Internal Combustion Engine, 2003, 32(6): 13-15.

[9] Z. J. Li, F. S. Liu, X. C. Bao. Modification Method of Heat Release Rate Calculation for Diesel Engine $[\mathrm{J}]$. Chinese Internal Combustion Engine Engineering, 2011, 32(5): 31-36, 43.

[10] T. L. Wang, Y. Ye, P. Ma, C. S. Zhao, L. Y. Kong. Experimental Investigation on Combustion Characteristics of Engine Fuelled with Ethanol-diesel Blends[J]. Journal of Chinese Agricultural Mechanization, Vol.35, Sep. 2014, pp. 141-143. 